\title{
Estudo colorimétrico de fritas feldspáticas
}

\section{(Colorimetric study of feldsphatic frits)}

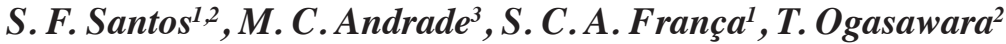 \\ ${ }^{I}$ Centro de Tecnologia Mineral - CETEM, Cidade Universitária, Av. Pedro Calmon 900 \\ Rio de Janeiro, RJ 21941-980 \\ ${ }^{2}$ Departamento de Engenharia Metalúrgica e Materiais, COPPE/UFRJ, CT/UFRJ, Bl. F, sala F210 \\ C. P. 68505, Cidade Universitária, Rio de Janeiro, RJ 21941-972 \\ ${ }^{3}$ Departamento de Química Analítica, IPRJ, R. Alberto Rangel s/n., Vila Nova \\ Nova Friburgo, RJ 28630-050 \\ ssantos@cetem.gov.br,shirleny@metalmat.ufrj.br
}

\begin{abstract}
Resumo
As fritas cerâmicas são materiais de natureza vítrea preparadas por fusão em temperaturas elevadas $\left(\sim 1500{ }^{\circ} \mathrm{C}\right)$, a partir de uma mistura de matérias-primas minerais. Os vidrados preparados exclusivamente a partir de fritas são utilizados principalmente em peças cerâmicas nas quais a componente estética é mais importante que a componente técnica, como no caso de azulejos, objetos decorativos e em restaurações odontológicas. Para um efeito decorativo mais eficiente, é necessário que o revestimento cerâmico seja estável o suficiente para não alterar significativamente a cor apresentada pelo pigmento e, dessa forma, possibilitar uma reprodutibilidade da cor obtida. Nesse contexto, este trabalho mostra um estudo de cor desenvolvida por pigmentos cerâmicos em fritas transparentes, obtidas a partir de feldspato da região Borborema-Seridó (PB/RN). A análise química do feldspato evidenciou que este mineral cumpre os requisitos necessários à aplicação como cerâmica (ou vidrado) de cobertura, pois apresenta teores mínimos de impurezas (minerais portadores de ferro e outros óxidos corantes) que deterioram a qualidade da frita obtida. O mineral foi caracterizado ainda por difração de raios X (albita e monoclínio) e quanto à granulometria (abaixo de $100 \mu \mathrm{m}$ ). A avaliação colorimétrica de acordo com os padrões CIElab e por medidas de refletância possibilitou caracterização da cor dos revestimentos obtidos em diferente fontes e iluminantes. Os resultados permitiram avaliar que o feldspato do Seridó apresenta potencial para aplicação como revestimento cerâmico colorido e futuramente poderia ser aplicado na área de restauração cerâmica odontológica, tendo em vista que esse mineral é um dos principais componentes das porcelanas odontológicas, porém, até o presente, todo material de restauração odontológica utilizado no Brasil é de origem estrangeira.
\end{abstract}

Palavras-chave: feldspato, fritas, CIElab, pigmentos cerâmicos.

\begin{abstract}
Ceramic frits are vitreous materials prepared by melting of minerals at high temperatures (about $1500{ }^{\circ} \mathrm{C}$ ). Glazes prepared exclusively from frits are used to manufacture ceramic pieces where the aesthetic component is more important than structural features, such as in the case of tiles, decorative ceramics and dental restorations. To improve the decorative effect the ceramic tile has to be colorimetrically stable in order to preserve the color provided by the pigment. In this context, the present work presents the results obtained in a color study carried out by application of ceramic pigments in transparent frits, produced with feldspar from Borborema-Seridó (PB/RN) region, Brazil. The chemical analysis of feldspar evidenced that this mineral present the requested characteristics for the application as glaze, once its impurities content (iron and other colorant oxides) are low grade. The mineral was characterized by $X$-ray diffraction (albite and orthoclase) and size distribution was determined by screening (Tyler sieves), as being less than 100 um. The colorimetric evaluation by using CIELab standards and reflectance measurements allowed characterizing the color of the ceramic tiles obtained under different light sources and illuminants. The results indicated that the feldspar from Serido presents potential for application as colored ceramic tile and hereafter it could be applied in the area of dental restorations, since that mineral is one of the main components of the dental porcelains, however, until the present, all dental restorative material used in Brazil is of foreign origin.
\end{abstract}

Keywords: feldspar, frits, CIElab, ceramic pigments.

\section{INTRODUÇÃO}

Os minerais estão exercendo uma influência cada vez maior sobre o desenvolvimento dos países. É possível observar a presença de grande quantidade de matérias- primas minerais em vários setores industriais, tais como: cerâmicos, vidros, cosméticos, isolantes, pigmentos, etc. Além disso, estudos recentes evidenciam que as rochas e os minerais industriais serão os recursos minerais mais importantes nos próximos séculos [1-3]. Na indústria 
mineral, os minerais são geralmente classificados em três classes: metálicos, não-metálicos e energéticos. A segunda classe pode ser subdividida em rochas e minerais industriais, gemas e águas minerais. Os minerais industriais podem ser aplicados diretamente como encontrados na natureza ou são utilizados como matéria-prima para fabricação de uma grande variedade de produtos [1]. Um importante ramo industrial que consome grande quantidade de minerais industriais é o setor cerâmico, onde se destaca o uso de feldspatos, argilas, caulim, quartzo, magnesita, cromita, grafita, dolomita, talco, etc [1]. Tomando como enfoque a produção de revestimentos cerâmicos (vidrados) e colorifícios, ressalta-se a importância do mineral feldspato, essencial para preparação destes produtos, e consumido abundantemente (cerca de $88 \%$ do feldspato beneficiado no Brasil) por estes setores $[4,5]$.

Feldspato, $\left[\mathrm{Na}, \mathrm{K}\left(\mathrm{AlSi}_{3} \mathrm{O}_{8}\right)\right]$, é o termo empregado para denominar um grupo de minerais constituídos de aluminossilicatos de potássio, sódio e cálcio. No Brasil os pegmatitos são a principal fonte de feldspato, com ocorrências principais na região nordeste (Paraíba e Rio Grande do Norte) e norte de Minas Gerais [4]. Nas indústrias de revestimentos cerâmicos é utilizado para fabricação de fritas, entre outras aplicações, atuando como fundente e promovendo uma vitrificação e transluzimento ao vidrado obtido como produto final [6]. Devido à estas importantes características ele é componente fundamental da porcelana feldspática utilizada em restaurações cerâmicas odontológicas, possibilitando o desenvolvimento de um aspecto similar ao do dente natural e propriedades de resistência à tensão que possibilitam seu uso no meio bucal sem complicações [7]. Nas restaurações odontológicas um pigmento cerâmico ou combinação de vários pigmentos é incorporado na composição protética. As camadas da restauração onde se concentram os pigmentos são o opaco, a dentina e o esmalte [7,8]. Contudo, a tarefa de colorir peças cerâmicas, incluindo as cerâmicas dentais, não é trivial. Além da necessidade de utilizar pigmentos que suportem as elevadas temperaturas e ataque químico, existem diversos fatores que contribuem para o desenvolvimento da cor e dificultam a correta caracterização e reprodutibilidade do tom obtido [9].

Um dos fatores que apresentam efeito notável sobre o desenvolvimento da cor de um objeto é a composição química da matriz. Isso é compreensível já que a composição dos vidros utilizados é bastante diversificada e o fato de que muitos óxidos estão presentes ao mesmo tempo na massa cerâmica tornam a tarefa de correlacionar resultados cromáticos com efeitos químicos muito complicados [10]. Outrossim, é preciso estabelecer uma metodologia adequada para caracterização das cores, uma vez que o método de avaliação visual que muitas indústrias ainda aplicam gera boa parte dos problemas em relação ao ajuste de cores [11].

Vale ressaltar que, embora no Brasil os feldspatos já sejam utilizados em vários setores, a aplicação em áreas avançadas, como em cerâmicas dentais, é ainda muito limitada. Logo, estudos científicos enfocando matériasprimas e produtos minerais são fundamentais e incentivam a agregação de valores a esses materiais. Este trabalho mostra resultados de estudos de caracterização colorimétrica de vidrados cerâmicos preparados com fritas obtidas a partir do feldspato da região Borborema-Seridó, no nordeste do Brasil.

\section{MATERIAIS E MÉTODOS}

\section{Sintese dos pigmentos de $\mathrm{Ce}_{1-x} \mathrm{Pr}_{x} \mathrm{O}_{2}$}

Para os testes colorimétricos das fritas utilizaramse pigmentos vermelhos baseado em óxidos de terrasraras $\left(\mathrm{Ce}_{1-\mathrm{x}} \mathrm{Pr}_{\mathrm{x}} \mathrm{O}_{2}\right)$, tendo em vista que o óxido de cério $\left(\mathrm{CeO}_{2}\right)$ já tem sido utilizado em odontologia para simular a fluorescência do esmalte do dente [12] e o composto $\mathrm{Ce}_{1-\mathrm{x}} \mathrm{Pr}_{\mathrm{x}} \mathrm{O}_{2}$ apresenta potencial para aplicação dental [13]. Por ser um método relativamente simples, a rota escolhida para síntese dos pigmentos foi o método Pechini modificado. Este método promove a formação de pós com boa homogeneidade, baixo tamanho de partícula (chegando a dimensões nanométricas), alta pureza, baixo custo e relativamente baixa temperatura de processamento [14]. A razão molar de ácido cítrico/metal foi fixada em $3 / 1$ e de ácido cítrico/etilenoglicol foi 2/1. A Fig. 1 ilustra o diagrama do procedimento utilizado para preparação dos pigmentos de $\mathrm{Ce}_{1-\mathrm{x}} \operatorname{Pr}_{\mathrm{x}} \mathrm{O}_{2}$, a caracterização química e mineralógica dos pigmentos está descrita [13].

Solução aquosa 0,2 molar de nitrato de cério hexahidratado (99\%, Sigma) foi adicionada lentamente a uma solução aquosa 1 molar de ácido cítrico (99,5\%, Vetec) aquecido a $70{ }^{\circ} \mathrm{C}$ sob agitação constante. Nas mesmas condições foi adicionada solução aquosa 0,05 molar de cloreto de praseodímio hexahidratado (99,9\%, Aldrich), variando-se a quantidade conforme o percentual de praseodímio desejado. Em seguida adicionou-se

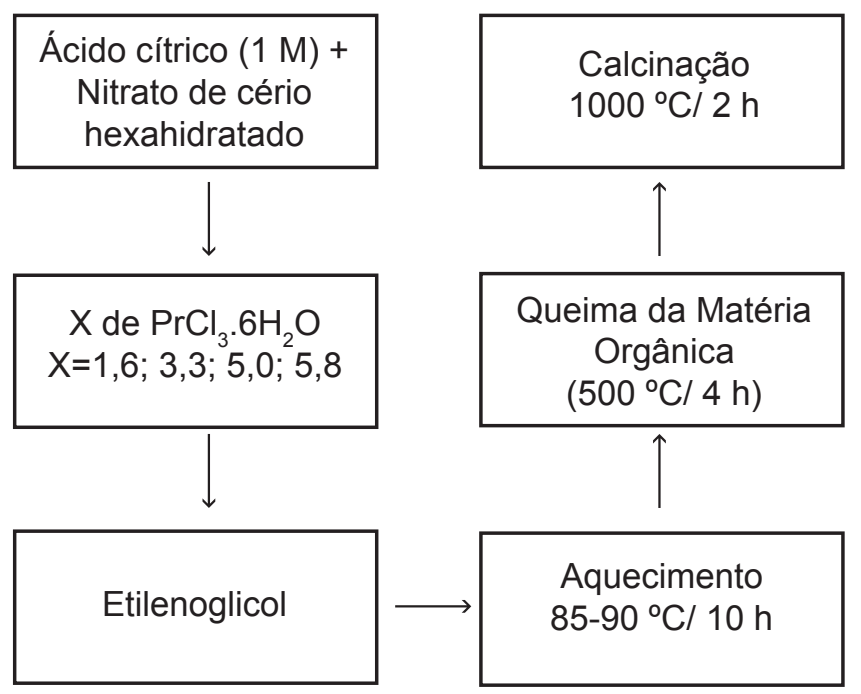

Figura 1: Fluxograma do procedimento utilizado para síntese dos pigmentos de $\mathrm{Ce}_{1-\mathrm{x}} \mathrm{Pr}_{\mathrm{x}} \mathrm{O}_{2}$.

[Figure 1: Flowchart of the synthesis process of $\mathrm{Ce}_{1-x} \mathrm{Pr}_{x} \mathrm{O}_{2}$ pigments.] 
etilenoglicol (99,5\%, Vetec). A temperatura foi elevada até $85-90{ }^{\circ} \mathrm{C}$ por $4 \mathrm{~h}$. Após este período obteve-se o complexo orgânico (polímero) resultante da reação de poliesterificação entre o poliálcool e as carboxilas livres dos sais complexos. Os polímeros foram tratados termicamente a $500{ }^{\circ} \mathrm{C}$ durante $4 \mathrm{~h}$, esfriados, desaglomerados e calcinados ao ar a $1000^{\circ} \mathrm{C}$ durante $2 \mathrm{~h}$. Em ambos os tratamentos térmicos a taxa de aquecimento foi $10^{\circ} \mathrm{C} / \mathrm{min}$ ao ar ambiente.

\section{Preparação da frita e dos revestimentos}

O feldspato utilizado neste trabalho é proveniente da região Borborema-Seridó nos limites dos estados da Paraíba e Rio Grande do Norte. Foram preparadas fritas cuja composição percentual em peso foi basicamente $80 \%$ de feldspato e $20 \%$ de bórax $\left(\mathrm{Na}_{2} \mathrm{~B}_{4} \mathrm{O}_{7} \cdot 10 \mathrm{H}_{2} \mathrm{O}, 99,5 \%\right.$, Vetec $)$. Esta mistura foi fundida a $1100{ }^{\circ} \mathrm{C}$ por $1 \mathrm{~h}$. Em seguida, a massa líquida foi bruscamente resfriada por imersão em água destilada e gelada. O material resultante foi moído até granulometria inferior a $44 \mu \mathrm{m}$. Obtiveram-se os revestimentos usando misturas de $2 \%$ em massa de pigmento para $98 \%$ numa massa de frita e $0,5 \mathrm{~mL}$ de solução aquosa contendo uma concentração mássica de 0,01\% de carboximetilcelulose (CMC 99,5\%, Synth). Estas misturas foram pinceladas sobre substratos de alumina densa $(3,5 \mathrm{~cm} \times 3,5 \mathrm{~cm})$ numa tentativa de simular a um dos tipos de material utilizado em cerâmicas dentais [13]. As placas recobertas foram secas a $100{ }^{\circ} \mathrm{C}$ durante $20 \mathrm{~min}$ e em seguida foram queimadas a $900^{\circ} \mathrm{C}$ (temperatura utilizada nos laboratórios de protéticos para recobrir a cerâmica dental) com taxa de aquecimento $10^{\circ} \mathrm{C} / \mathrm{min}$ por $30 \mathrm{~min}$.

\section{Técnicas de caracterização}

Difração de raios X: a estrutura cristalográfica dos pós foi determinada em um difratômetro Bruker-AXS D5005, radiação Co-Ka (35 kV/40 mA), coletado de 5 a $80^{\circ} \mathrm{em}$ 20. A interpretação qualitativa de espectro foi efetuada por comparação com padrões contidos no banco de dados PDF02 (ICDD, 1996) em software Bruker DiffracPlus.

Espectrometria de fluorescência de raios X: a composição química das amostras foi verificada por fluorescência de raios $\mathrm{X}$ em um equipamento $\mathrm{S} 4$ Bruker, por método da pastilha prensada e varredura semiquantitativa.

Colorimetria: foram adquiridas coordenadas colorimétricas utilizando o método CIELab [9]. O método CIELab (Commission Internationale de L'Eclairage) permite medir a intensidade de absorção na região visível para obtenção dos parâmetros $\mathrm{L}^{*}$, referente à luminosidade que varia do negro (0) ao branco (100), a* que é intensidade de cor vermelho(+)/ verde(-) e b* a intensidade de cor amarelo(+)/azul(-) [15]. A reflectância na região do visível e as coordenadas colorimétricas dos materiais obtidos foram medidas usando colorímetro Color Touch, sob ação de fonte D65 num ângulo de $10^{\circ}$ e alguns iluminantes (D65, C, A, F). O termo fonte de luz refere-se à luz real que atinge a amostra, enquanto os iluminantes são o resultado de uma distribuição espectral que simula condições de iluminação comuns em nosso dia-a-dia [15].

\section{RESULTADOS E DISCUSSÃO}

\section{Caracterização do feldspato e da frita}

A composição química do feldspato está na Tabela I. Foi possível verificar a presença de baixos teores de impurezas, isto é, dos minerais portadores de ferro e outros óxidos corantes, que poderiam deteriorar a qualidade do produto [5]. Desta forma, a frita obtida mostrou-se totalmente transparente antes do pigmento ser introduzido na composição, garantindo assim maior acuidade dos resultados de cores obtidos, como será discutido posteriormente.

Tabela I - Composição química mássica do feldspato utilizado na preparação da frita.

[Table I - Chemical composition of the feldspar used in the preparation of the frit.]

\begin{tabular}{cccc}
\hline Compostos & $\begin{array}{c}\text { teor } \\
(\%)\end{array}$ & Compostos & $\begin{array}{c}\text { teor } \\
(\%)\end{array}$ \\
\hline $\mathrm{SiO}_{2}$ & 66,0 & $\mathrm{~K}_{2} \mathrm{O}$ & 5,5 \\
$\mathrm{Al}_{2} \mathrm{O}_{3}$ & 20,0 & $\mathrm{Rb}_{2} \mathrm{O}$ & 0,07 \\
$\mathrm{CaO}$ & 0,26 & $\mathrm{NiO}$ & 0,11 \\
$\mathrm{Na}_{2} \mathrm{O}$ & 5,9 & $\mathrm{Cinzas}$ & 1,3 \\
$\mathrm{P}_{2} \mathrm{O}_{5}$ & 0,34 & - & - \\
\hline
\end{tabular}

A análise por difração de raios $\mathrm{X}$ indicou que este feldspato é uma mistura de albita (feldspato sódico) e monoclínio (feldspato potássico), Fig. 1, presentes quase na mesma proporção, como avaliado na Tabela I pela relação entre os teores de $\mathrm{Na}_{2} \mathrm{O}$ e $\mathrm{K}_{2} \mathrm{O}$. De acordo com os diagramas de fase correspondentes, esta característica singular faz com que este feldspato apresente uma temperatura de fusão de $\sim 1200{ }^{\circ} \mathrm{C}$. Neste trabalho foi possível aplicar uma temperatura ainda menor $\left(1100^{\circ} \mathrm{C}\right)$ devido à incorporação de bórax (fundente) à composição [16]. O feldspato foi moído até granulometria inferior a $100 \mu \mathrm{m}$ para ser utilizado na preparação da frita, pois esta é a faixa granulométrica mínima necessária para que todo material seja fundido

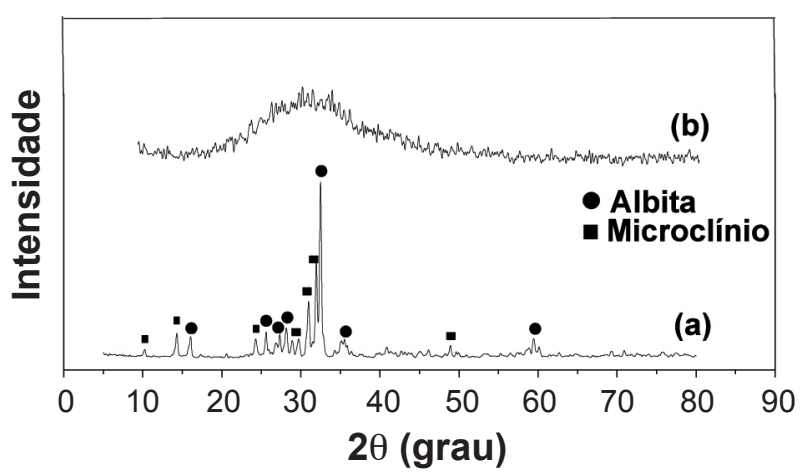

Figura 2: Difratogramas de raios X de feldspato (a) e frita (b). [Figure 2: X-ray diffraction patterns of the feldspar (a) and the frit $(b)$.] 
durante o tempo de permanência no forno. Caso contrário, defeitos podem ocorrer (como presença de fases não fundidas) no produto final [5].

A análise por difração de raios $\mathrm{X}$ da frita feldspática, (Fig. 2), mostrou um perfil característico da formação de materiais integralmente vítreos caracterizado pelo halo centrado em torno de $2 \theta=27^{\circ}$, sem fases cristalinas remanescentes do feldspato original [17].

\section{Caracterização colorimétrica dos revestimentos}

As coordenadas colorimétricas para os pigmentos de $\mathrm{Ce}_{1-\mathrm{x}} \operatorname{Pr}_{\mathrm{x}} \mathrm{O}_{2}$ são mostradas na Tabela II. A análise geral dos dados evidenciou o predomínio de valores de $\mathrm{a}^{*} \mathrm{e} \mathrm{b}^{*}$ positivos, que no sólido de cores localiza os pigmentos na região da tonalidade vermelha, que corresponde ao aspecto dos pós a olho nu [18]. É possível observar também uma tendência geral para diminuição dos valores de $\mathrm{L}^{*} \mathrm{e}$ aumento dos valores de $\mathrm{a}^{*}$ devido à saturação do tom vermelho à medida que se aumenta a quantidade de praseodímio na rede cristalina do $\mathrm{CeO}_{2}[19]$.

Os parâmetros colorimétricos obtidos para os revestimentos vidrados aos quais foram incorporados os pigmentos de $\mathrm{Ce}_{1-\mathrm{x}} \operatorname{Pr}_{\mathrm{x}} \mathrm{O}_{2}$ são também mostrados na Tabela II. Observou-se em todos os casos um aumento da luminosidade e pequena diminuição da coordenada $a^{*}$, devido ao fato de que o pigmento está disperso numa matriz vítrea transparente que dilui o tom e reflete a luminosidade da própria matriz [9]. No entanto observou-se um aumento significativo da coordenada $\mathrm{b}^{*}$, indicando deslocamento no sentido da tonalidade amarela. A análise da composição da frita evidenciou que nenhum dos compostos presentes poderia favorecer uma interferência de cor [10]. Entretanto, encontram-se na literatura registros de que a presença de $\mathrm{CeO}_{2}$ em vitro-cerâmicas poderia favorecer o desenvolvimento de um tom marfim, neste caso um tipo de interação similar poderia estar ocorrendo entre o pigmento de $\mathrm{Ce}_{1-\mathrm{x}} \mathrm{Pr}_{\mathrm{x}} \mathrm{O}_{2}$ e a matriz vítrea $[20,21]$.

Os pigmentos e os revestimentos obtidos foram ainda caracterizados por medidas de refletância, Fig. 3 .

$\mathrm{O}$ perfil observado para os pigmentos de $\mathrm{Ce}_{1-x} \mathrm{Pr}_{x} \mathrm{O}_{2}$ é caracterizado por uma banda com forte absorção na faixa 400-650 nm, correspondente ao desenvolvimento da cor vermelha, intensificada com a incorporação de pigmentos com maior conteúdo de praseodímio [15]. Verificouse, contudo, uma modificação do formato da banda nos

Tabela II - Valores de $\mathrm{L}^{*}, \mathrm{a}^{*} \mathrm{e} \mathrm{b}^{*}$ para os pigmentos de $\mathrm{Ce}_{1-\mathrm{x}} \operatorname{Pr}_{\mathrm{x}} \mathrm{O}_{2}$ e revestimentos coloridos.

[Table II - Colorimetric Coordinates ( $L^{*}, a^{*}$ and $\left.b^{*}\right)$ for pigments of $C e_{1-x} \operatorname{Pr}_{x} \mathrm{O}_{2}$ and for colored coatings.]

\begin{tabular}{cccccccc}
\hline Pigmentos & $\mathrm{L}^{*}$ & $\mathrm{a}^{*}$ & $\mathrm{~b}^{*}$ & Revestimentos & $\mathrm{L}^{*}$ & $\mathrm{a}^{*}$ & $\mathrm{~b}^{*}$ \\
\hline $\mathrm{Ce}_{0,984} \mathrm{Pr}_{0,016} \mathrm{O}_{2}$ & 66,82 & 18,46 & 19,70 & $\mathrm{R} \mathrm{Ce}_{0,984} \mathrm{Pr}_{0,016} \mathrm{O}_{2} .2 \%$ & 74,13 & 17,85 & 29,03 \\
$\mathrm{Ce}_{0,967} \mathrm{Pr}_{0,033} \mathrm{O}_{2}$ & 54,74 & 21,58 & 17,74 & R.Ce $_{0,967} \mathrm{Pr}_{0,033} \mathrm{O}_{2} .2 \%$ & 76,00 & 16,74 & 27,12 \\
$\mathrm{Ce}_{0,950} \mathrm{Pr}_{0,050} \mathrm{O}_{2}$ & 44,50 & 23,72 & 16,50 & R.Ce $_{0,950} \operatorname{Pr}_{0,050} \mathrm{O}_{2} .2 \%$ & 68,45 & 22,05 & 32,52 \\
$\mathrm{Ce}_{0,942} \mathrm{Pr}_{0,058} \mathrm{O}_{2}$ & 39,30 & 24,76 & 17,27 & R.Ce ${ }_{0,942} \operatorname{Pr}_{0,058} \mathrm{O}_{2} .2 \%$ & 67,72 & 22,43 & 32,48 \\
\hline
\end{tabular}
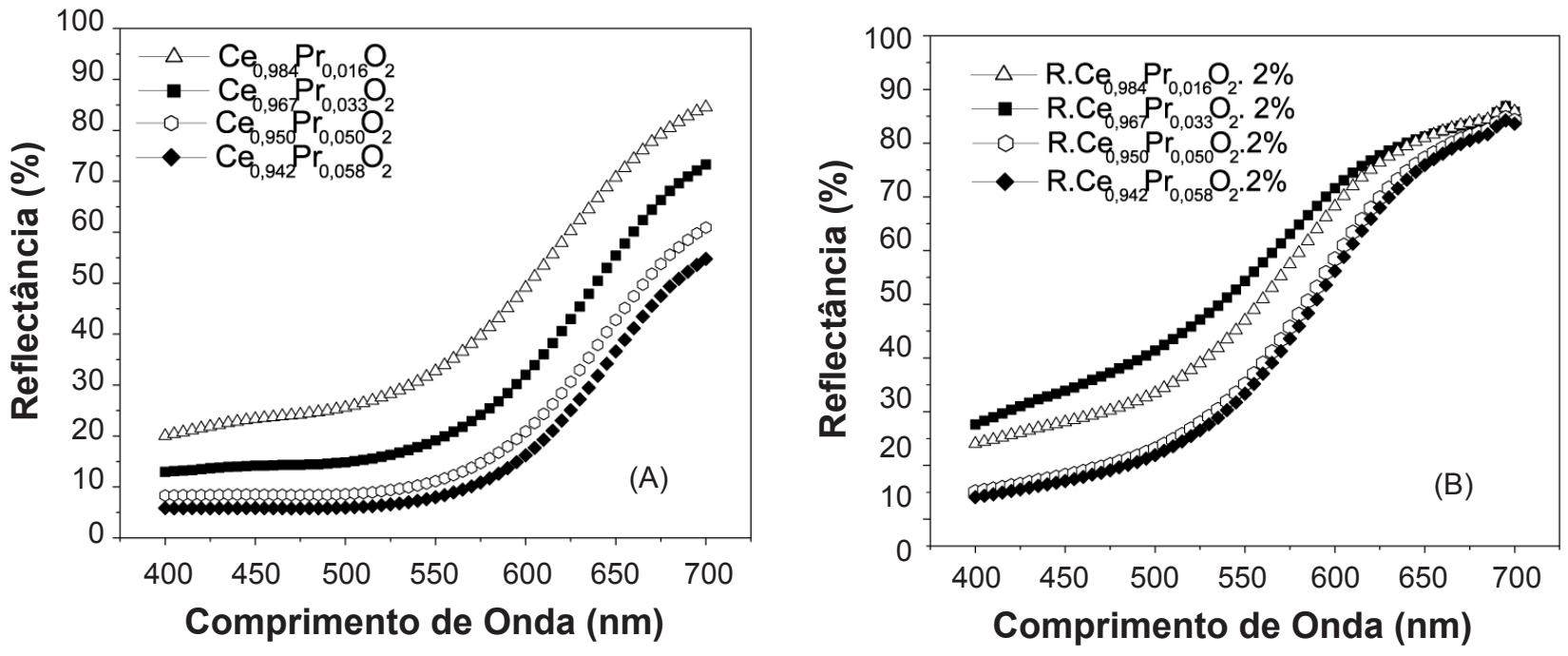

Figura 3: Curva de refletância de pigmentos de $\mathrm{Ce}_{1-x} \operatorname{Pr}_{x} \mathrm{O}_{2}$ (a) e revestimentos coloridos com $\mathrm{Ce}_{1-\mathrm{x}} \operatorname{Pr}_{\mathrm{x}} \mathrm{O}_{2}$ (b). [Figure 3: Reflectance curves of the $\mathrm{Ce}_{1-x} \mathrm{Pr}_{x} \mathrm{O}_{2}$ pigments( $(a)$ and for colored coating $\left.(b).\right]$ 
Tabela III - Valores de $\mathrm{L}^{*}, \mathrm{a}^{*} \mathrm{e} \mathrm{b}^{*}$ para os revestimentos sob ação de vários iluminantes. [Table III - Colorimetric coordinates $\left(L^{*}, a^{*}\right.$ and $\left.b^{*}\right)$ for the coatings in different illuminants.]

\begin{tabular}{|c|c|c|c|c|c|}
\hline Iluminantes & & R.Ce ${ }_{0,984} \mathrm{Pr}_{0,016} \mathrm{O}_{2} .2 \%$ & R.Ce ${ }_{0,967} \mathrm{Pr}_{0,033} \mathrm{O}_{2} .2 \%$ & $\begin{array}{c}\text { R.Ce } e_{0,950} \mathrm{Pr}_{0,050} \mathrm{O}_{2} \cdot 2 \\
\end{array}$ & R.Ce ${ }_{0,942} \mathrm{Pr}_{0,058} \mathrm{O}_{2} .2 \%$ \\
\hline \multirow{3}{*}{ D $65^{1}$} & $\mathrm{~L}^{*}$ & 74,13 & 76,00 & 68,45 & 67,72 \\
\hline & $a^{*}$ & 17,85 & 16,74 & 22,05 & 22,43 \\
\hline & $b^{*}$ & 29,03 & 27,12 & 32,52 & 32,48 \\
\hline \multirow{3}{*}{$\mathrm{C}^{2}$} & $\mathrm{~L}^{*}$ & 74,22 & 76,08 & 69,07 & 67,84 \\
\hline & $a^{*}$ & 16,70 & 15,64 & 20,78 & 21,16 \\
\hline & $b^{*}$ & 29,28 & 27,35 & 32,81 & 32,78 \\
\hline \multirow{3}{*}{$\mathrm{F}^{3}$} & $\mathrm{~L}^{*}$ & 74,26 & 76,14 & 69,05 & 67,80 \\
\hline & $a^{*}$ & 15,23 & 14,26 & 19,07 & 19,43 \\
\hline & $b^{*}$ & 29,60 & 27,65 & 33,03 & 32,97 \\
\hline \multirow{3}{*}{$\mathrm{A}^{4}$} & $\mathrm{~L}^{*}$ & 77,84 & 79,50 & 73,28 & 72,08 \\
\hline & $\mathbf{a}^{*}$ & 21,71 & 20,32 & 25,97 & 26,41 \\
\hline & $\mathbf{b}^{*}$ & 34,41 & 32,27 & 38,93 & 38,95 \\
\hline
\end{tabular}

${ }^{1}$ luz do dia; ${ }^{2}$ luz solar difusa de um céu nublado ao meio dia; ${ }^{3}$ luz de uma fonte fluorescente fria; ${ }^{4}$ lâmpada incandescente.

revestimentos coloridos provavelmente devido à influência do tom amarelado desenvolvido pelo vidrado, conforme havia sido observado na análise das coordenadas colorimétricas [22]. Todavia, há que se levar em consideração também a absorção advinda da presença da própria matriz vítrea e o fato de que a cor vermelha dos pigmentos foi preservada, ou seja, ocorreu o surgimento de um leve tom amarelado, no entanto esta cor não interagiu com os pigmentos de maneira a modificar significativamente a cor obtida.

A Tabela III exibe os valores de coordenadas colorimétricas dos quatros revestimentos coloridos sob ação de vários iluminantes, que reflete a constância da cor em diferentes condições de luminosidade encontradas no dia-a-dia. Sabe-se que idealmente a diferença de cor entre revestimentos submetidos a diferentes iluminantes não deve exceder 5 unidades de CIElab (na análise individual de cada parâmetro $-\mathrm{L}^{*}, \mathrm{a}^{*}, \mathrm{~b}^{*}$ ) para que a diferença não altere a boa percepção de cor da peça [10].

Foi possível observar que os revestimentos apresentaram boa constância das cores, com exceção da ação sobre o iluminante $\mathrm{A}$, que apresentou alguns valores maiores do que 5 de diferença em relação ao padrão (D65, luz do meio dia), no entanto esta diferença pode ser considerada aceitável, dependendo do tipo de aplicação, pois este iluminante representa a condição de luminosidade de uma sala fechada, onde é comum que ocorram distorções na aparência dos objetos [15]. Há uma complexidade de fatores que contribuem para o desenvolvimento da cor. Apesar disto, os resultados discutidos exibiram uma avaliação colorimétrica que evidenciou a boa qualidade apresentada pelo feldspato da região Borborema-Seridó em relação à introdução de pigmentos. Além disso, levando-se em conta o fato de que o crescimento do interesse por determinada matéria-prima está vinculado também à quão bem caracterizado ele está tecnicamente, estes resultados podem contribuir para uma ampliação do campo de aplicação deste mineral.

\section{CONCLUSÕES}

Os revestimentos coloridos obtidos a partir do feldspato da região Borborema-Seridó e do pigmento de $\mathrm{Ce}_{1-\mathrm{x}} \operatorname{Pr}_{\mathrm{x}} \mathrm{O}_{2}$ apresentaram coloração vermelha com saturação do tom à medida em que aumentava a concentração de praseodímio nos pigmentos incorporados. Foi possível verificar ainda a constância das cores dos revestimentos sob ação de diferentes iluminantes, em que observaram-se desvios somente sob ação do iluminante A. O feldspato da região BorboremaSeridó mostrou potencial para aplicação em cerâmicas de cobertura com características similares às cerâmicas dentais, pois embora uma alteração no sentido da tonalidade amarela tenha sido observada, a cor vermelha dos pigmentos não foi significativamente alterada.

\section{REFERÊNCIAS}

[1] A. B. Luz, J. A. Sampaio, S. L. M. Almeida, Tratamento de Minérios, $4^{\text {a }}$.ed., CETEM/MCT, Rio de Janeiro, RJ (2004) 10.

[2] A. B. Macedo, Estudos Avançados 12, 33 (1998) 67.

[3] L. Horowitz, J. Cleaner Production 14, 3/4 (2006) 307.

[4] A. B. Luz, F. F. Lins, Rochas e Minerais Industriais: Usos e Especificações, $1^{a}$ Ed., CETEM/MCT, Rio de Janeiro, RJ 
(2005) 413.

[5] E. Sánches, Cerâmica Industrial 2, 3/4 (1997) 32.

[6] R. N. Almeida, S. F. Santos, J. A. Sampaio, A. B. Luz, T. Ogasawara, M. C. Andrade, Cerâmica 53, 325 (2007) 57.

[7] C. Panzera, L. M. Dimeglio, C03C 10/10, US Patent $5,653,791$ (1997).

[8] A. G. Wee, W. Y. Chen, W. M. Johnston, Dental Mater. 21, 7 (2005) 665.

[9] F. G. Melchiades, A. O. Boschi, Cerâmica Industrial 4, 1 (1999) 1.

[10] P. Zannini, Colore, Pigmenti e Colorazione in Ceramica, $1^{\text {a }}$ Ed, Società Ceramica Italiana, Modena, Italia (2003) 59.

[11] P. P. Machado, D. Hotza, C. Petter, C. P. Bergmann, Cerâmica Industrial 2, 3/4 (1997) 51.

[12] W. J. O’brien, Dental Mater. 14, 14 (1998) 365.

[13] S. F. Santos, M. C. Andrade, J. A. Sampaio, A. B. Luz, T. Ogasawara, Dyes and Pigments 75, 3 (2007) 574.

[14] R. A. Candeia, M. I. B. Bernardi, A. G. de Souza, E. L. da Silva, I. M. G. Santos, Mater. Lett. 58, 5 (2004) 569.

[15] J. Popson, D. D. Malthouse, Measurement and Control of the Optical Properties of Paper, $2^{\text {nd }}$ Ed., $9^{\text {th }}$ Publ. Copyright, New Albany, EUA (1996).

[16] N. L. Weiss, Mineral Processing Handbook, $1^{\text {st }}$ Ed., Society of Mining Engineers, New York, EUA (1985).

[17] D. A. Earl, D. E. Clark, Cerâmica Industrial 6, 1 (2001) 43.

[18] P. Sulcová, M. Trojan, Z. Solc, Dyes Pigments 37, 1 (1998) 65

[19] S. T. Aruna, S. Ghosh, K. C. Patil, Int. J. Inorganic Mater. 3, 4-5 (2001) 387.

[20] G. Yoldjian, J. Less Common Metals 111, 1-2 (1985) 17.

[21] Y. Sun, Z. Wang, J. Tian, X. Cao, Mater. Lett. 57, 2 (2002) 425 .

[22] P. Sulcová, Dyes Pigments 47, 3 (2000) 285.

(Rec. 21/11/2008, Rev. 29/01/2009, Ac.03/07/2009) 